\title{
La santé publique et la violence des proportions dans le roman. Autour de Heather O'Neill et Rawi Hage.
}

\author{
Daniel Laforest \\ Université de l'Alberta
}

\begin{abstract}
Résumé
Cet article se penche sur les rapports croisés entre la littérature, la médecine, et le concept de santé en vertu de l'hypothèse voulant que l'indicible traditionnellement associé à l'expérience des confins de la maladie et de la douleur soit une notion construite par la littérature et qui n'est opérante qu'à l'intérieur de ses systèmes de signification et d'assignation de valeurs. La médecine est au contraire posée comme un système producteur de sens où l'indicible n'a pas cours. Cela dit, un examen des croisements contemporains entre littérature et médecine révèle que l'idée d'une violence contraignant la subjectivité au silence ou à l'a-sémantisme s'est en réalité déplacée et exerce désormais son influence dans un autre type de rapports : entre la parole littéraire et le système de santé en tant « qu'objet » bien réel mais dont la visibilité n'est envisageable qu'à travers ses effets. Les romans Lullabies for Little Criminals de Heather O'Neill et Cockroach de Rawi Hage sont brièvement analysés en guise d'exemples.
\end{abstract}

\begin{abstract}
This article examines the relations between literature, medicine, and the concept of health based on the hypothesis that the notion of " ineffable » which is usually associated with extreme experiences of illness or pain is rather produced by literature itself, proving efficient solely within the confines of its systems of meaning-making and value-attribution. Conversely, medicine is posited as a meaning-making system where the idea of the ineffable is irrelevant. However, an examination of contemporary intersections between literature and medicine reveals that what is traditionally seen in literature as an ontological violence capable of reducing the subject to silence or a-significance has become tantamount to a new type of rapports. These rapports are now situated between the literary discourse and the healthcare system, with the latter appearing as an " hyper-object » whose real existence can only be seized via its effects. The novels Lullabies for Little Criminals by Heather O'Neill and Cockroach by Rawi Hage are briefly examined in order to illustrate that.
\end{abstract}



Ouvrons ce texte avec une petite fable. Une fable, qui comme la plupart des fables modernes, est issue d'un fait divers apparemment bien réel relayé dans les médias. Je garde le souvenir d'avoir lu, enfant, un entrefilet dans le quotidien de ma ville à propos d'un bibliothécaire américain excentrique, ou alors trop féru de science-fiction, qui avait émis le souhait de faire congeler sa tête à sa mort. Il spécifiait que cette partie unique de son corps suffirait, et ne manifestait pas d'intérêt pour la préservation de ses autres membres ou organes. Son raisonnement était qu'une société future serait apte, en vertu des progrès technologiques, à décongeler sa tête et à lui redonner une seconde existence, peu importe la façon précise. Cela dit, son but n'était pas la vie éternelle. Je rappelle qu'il était bibliothécaire. Son seul désir, qui faisait le sel de l'anecdote, était de se garantir la chance de lire tous les livres qu'il n'aurait pas eu le temps de lire dans sa première vie. Il souhaitait donc une seconde vie mais seulement pour ses yeux et son cerveau, suivant l'idée que la lecture n'implique pas les autres parties du corps et de l'organisme. Comme je l'ai suggéré, mon souvenir de cet entrefilet, avec le temps, s'est transformé en souvenir d'une fable exemplaire. Pourquoi ? Parce que plus tard au fil de mes propres lectures j'ai pu me dire : mais cette histoire au fond, c'est celle de Joé Bousquet, ce jeune soldat français paralysé à la Première Guerre et qui, alité au milieu des livres et en proie à de grandes souffrances physiques, consacra le reste de ses jours à une œuvre littéraire admirée par les surréalistes, par André Gide, et surtout par la philosophe 
Simone Weil qui en fit un exemple incarné du triomphe de l'esprit (Weil, 1999, p. 661-670 et p. 791-800). Mais cette histoire, c'est aussi celle de Tony Judt, historien britannique d'origine juive que la maladie de Lou Gehrig (ALS) confina à l'immobilité dans les deux dernières années de sa vie et qui se décrit dans ses mémoires publiées après sa mort sous le titre The Memory Chalet en train de «parcourir sa vie, ses pensées, ses fantasmes, ses souvenirs véritables ou fabriqués, jusqu'à ce qu'il trouve les événements, les personnes, ou les histoires en mesure de distraire son esprit de corps dans lequel il est emprisonné. » (Judt, 2010, p. 19) Dans la fiction enfin, cela fait immanquablement penser au personnage tragique du roman Johnny Got His Gun de Dalton Trumbo publié en 1939, et qui fit l'objet d'une adaptation remarquée au cinéma réalisée par l'auteur lui-même en 1971. Un jeune soldat dont le corps quasi pulvérisé par un obus se retrouve sur un lit d'hôpital privé de ses bras, de ses jambes, et d'à peu près tout son visage, est incapable de communiquer avec l'extérieur mais n'a rien perdu de sa faculté de penser. Enfin c'est toute la controverse provoquée par la sortie publique en 2015 du neurochirurgien italien Sergio Canavero clamant son désir de réaliser incessamment une première greffe de tête réussie (St-Germain, 2017). Dans chacune de ses occurrences cette fable en est une qui, en définitive, nous montre une pensée complètement et drastiquement séparée du fonctionnement corporel.

\section{Un triomphe limité de la littérature face à la médecine}

C'est sur le lien souvent étroit avec les livres et la lecture que l'on doit s'arrêter ici. À travers ce lien notre fable représente assurément un triomphe de la littérature. Mais il ne s'agit pas, comme un premier élan nous porterait à le croire, d'un triomphe de la littérature sur la mort. La littérature ou du moins la narrativité encore accessible aux corps immobilisés, meurtris et souffrants dans le paradigme résumé par notre fable est plutôt ce qui l'emporte sur la 
médecine. En effet si nous entendons la médecine comme la discipline qui vise le maintien de l'équilibre et de l'intégrité du corps, alors ceux qui comme Bousquet, Judt et plusieurs autres ont lu et composé des livres en dépit de la mise en échec de leur capacité motrice, et en faisant fi des souffrances afférentes, nous apparaissent comme les porteurs du triomphe de la pensée sur les contraintes du corps et de la biologie. Ce triomphe court sur plusieurs siècles. Homère l'aveugle, Virginia Woolf la neurasthénique, William Faulkner l'alcoolique, les philosophes Gilles Deleuze et Jean-Luc Nancy sujets respectivement de l'ablation d'un poumon et d'une transplantation cardiaque, l'astrophysicien et vulgarisateur Stephen Hawking en fauteuil roulant et s'exprimant par le biais d'un clavier : Toutes ces figures ont pensé et écrit malgré tout, c'est-à-dire qu'elles ont fait œuvre d'esprit malgré la violence faite à leur corps.

Mais la médecine et la littérature n'existent jamais seule. Quand un corps et une subjectivité en font la rencontre, c'est à chaque fois à l'intérieur d'un cadre systémique. Le bibliothécaire espérant faire congeler sa tête aimait de toute évidence lire énormément de livres. Mais ce n'est pas cela qui définit la mégalomanie de son projet, c'est la prétention de s'octroyer plus qu'une vie dans l'espoir de lire un jour toute la littérature. Personne ne lit ni ne lira jamais toute la littérature puisque 'littérature' est le nom du système d'assignation de sens et d'appartenances, de rapports de forces et d'influences, enfin de tout ce qui concourt à faire que des textes soient considérés à l'intérieur d'un système plutôt que d'un autre. Idem pour la médecine qui est le nom de la discipline et de son épistémologie en tant qu'elles existent et s'exercent à l'intérieur de ce qu'on appelle désormais couramment un système de santé. Voilà donc ce qui nous occupera au premier chef dans ce qui suit : le problème de la proportion dans le rapport entre la littérature et la médecine. Les écrivains dont le corps s'est 
www.revue-analyses.org, vol. 14, n², automne-hiver 2019, p. 20-37.

abîmé avant leur esprit et leur créativité ont continué à écrire des livres malgré leurs épreuves médicales. C'est là un premier niveau de formalisation. Le second niveau consiste à dire que dans chacune de ces occurrences la littérature a prévalu sur la gestion de la santé. Les deux niveaux expriment la même idée. Mais ils demeurent séparés par une différence proportionnelle qu'on supposera difficilement surmontable. C'est ce problème que l'on examinera dans les pages qui suivent. En se faisant système de santé la médecine contemporaine est devenue le lieu de nouveaux rapports proportionnels qui bouleversent le rapport de la violence et du corps, et par conséquent le rapport de soi à soi. Qu'en est-il de la littérature et de ses imaginaires dans cette équation ? La littérature est confrontée à une nouvelle question, qui est peut-être, aussi, une nouvelle forme de violence pour elle : Existet-il des objets liés à la santé, des corps qui, simplement de par leurs proportions, sont insaisissables pour la pensée littéraire ? L’objectif d'une telle problématique est de proposer un modèle de lecture alternatif où se trouverait amoindrie la démarcation qui maintient chacune dans leur camp la littérature et la médecine.

Dire comme plus haut que des écrivains continuent leur œuvre malgré la violence faite à leur corps par l'accident ou la maladie chronique consiste à créer une ligne de séparation entre deux formes de violence subie par le corps : celle qui est considérée comme dicible, et celle qui ne l'est pas. C'est un modèle bien commode pour la littérature. En effet la littérature, la philosophie ou l'œuvre scientifique affranchie du corps physique de l'écrivain devient comme une grande aventure de la vie intérieure et c'est pourquoi elle peut en quelque sorte s'extrapoler elle-même pour dire presque toutes les formes de violence : l'abject, le monstrueux, l'inhumain. Or il faut bien dire « presque », car il y aurait cette autre violence qui commencerait lorsque la parole, l'imagination, et la faculté de conceptualisation 
ne sont plus possibles : la douleur extrême, sans lieu d'assignation spécifique sur le corps et qui confine à l'agonie. Cette violence marque la limite du dicible pour la littérature car l'écrivain avouera ne pas pouvoir l'endosser légitimement dans l'imagination sans l'avoir expérimentée dans la réalité. Cette douleur offre un cas d'exception où la pensée et la maladie se fondent l'une dans l'autre. Et c'est une exception qui confirme en quelque sorte la règle précédente puisqu'elle se voit réservée à des individus rares dont l'expérience est alors perçue comme celle d'un explorateur des confins de l'expérience humaine plutôt que celle d'un simple malade. Témoin encore Simone Weil écrivant à Joé Bousquet : « vous êtes infiniment privilégié, car vous avez la guerre logée à demeure dans votre corps. » (Weil, 1999, p. 794) Ou plus près de nous, le philosophe Ruwen Ogien se posant la question de savoir si l'on peut encore pratiquer la philosophie au sens traditionnel du terme quand on est à son instar atteint d'un cancer et qu'on subit des traitements massifs pour l'endiguer (Ogien, 2017). On pourrait même choisir de voir cela réuni dans le courant marginal quoiqu'indéniable des écrivains qui comme William Styron dans son célèbre Darkness Visible (1990) ont pris la plume pour essayer de raconter la dépression dont ils ont été affligés, et qui ont tous reconnu comme point de départ le paradoxe voulant que la nature même de cette maladie consiste dans son inadéquation avec le langage et la narration ${ }^{1}$.

\section{L'idée de proportion comme antidote à l'indicible}

Cela dit la réflexion resterait incomplète à s'arrêter sur ce dernier point. Il faut voir que cet indicible de la violence qui habite le corps jusque dans sa substance la plus intime, il n'existe en fait que pour la littérature. Parce que sinon, il n'a plus rien d'indicible, et encore moins

\footnotetext{
${ }^{1}$ Nel Casey (ed.), Unholy Ghost : Writers on Depression, New York Perennial, 2002 ; Andrew Solomon, The Noonday Demon : An Atlas of Depression, New York, Scribner, 2001.
} 
www.revue-analyses.org, vol. 14, n², automne-hiver 2019, p. 20-37.

d'ineffable. La blessure et la souffrance capables par leur intensité de dépasser le langage, dès lors qu'on les considère en-dehors du système de la littérature, deviennent parties prenantes de l'univers du « médical» et plus largement de celui de la gestion de la santé. C'est-à-dire qu'elles appartiennent désormais à un monde de science et de technique où, croit-on, il convient d'agir sans parler, et de ne jamais parler pour rien dire. La violence qui paraissait indépassable dans le domaine de la littérature et de l'imagination devient sinon bénigne, du moins gérable quand elle on l'appréhende à partir de la médecine. Le cancéreux « combat» son cancer ; le dépressif est enjoint d'essayer de «mettre à distance » sa dépression, de se séparer d'elle. Nous sommes alors en phase avec la définition de la violence par la phénoménologie française contemporaine qui nous dit que pour la penser correctement nous n'avons pas le choix de la concevoir en-dehors de ce qu'elle affecte : « [la violence est] mise en œuvre d'une force qui reste étrangère au système dynamique ou énergétique dans lequel elle intervient. » (Nancy, 2003, p. 36) Avec la littérature et la médecine, il y a donc deux modèles complémentaires où sont renvoyées face à face une violence qui se dit et une violence qui ne se dit pas. Le premier modèle confère à la littérature tous pouvoirs de parole sauf celui de dire la souffrance extrême qui réduit au mutisme le corps qui l'éprouve. Mais le second modèle atténue quant à lui cette amplitude du pathos. La médecine prend le relais de la littérature, dirait-on, quand elle prend acte de la souffrance du corps et cherche à agir sur elle sans un recours nécessaire au langage qui en exprimerait les milles détails et variations. L'indicible qui arrête le mouvement de la littérature ne pose donc pas de problème particulier pour la médecine. L'idée qui remonte aux Grecs mais qui a connu une recrudescence ces dernières années en France, voulant que la littérature puisse viser un rôle 
« thérapeutique ${ }^{2} »$, est révélatrice de cet état de fait dans sa tentative avouée de renverser la dynamique entre ces deux plans de la violence du dicible littéraire et de l'indicible médical. Révélatrice car elle met plus que jamais leur démarcation en lumière, et elle en souligne l'importance pour comprendre notre époque.

La violence n'a peut-être pas lieu, comme on le pense souvent, entre la littérature et le monde réel, au sens où il s'agirait là de deux plans tout à fait distincts réunis seulement un système de références ou d'équivalences par la convention arbitraire du langage. C'est ici que la notion théorique de proportion devient opérante. La violence n'a peut-être pas lieu entre les mots sur la page et leur référent dans l'esprit. Elle n'a sans doute pas lieu seulement, non plus, entre le point fermé et la gueule qui se le prend dans le réel. La violence telle qu'elle nous intéresse ici — c'est-à-dire en tant qu'elle oscille entre le dicible et l'indicible — a d'abord lieu dans les proportions exprimées dans chacun de ces rapports. Et en un second temps, elle a aussi lieu dans les proportions qui s'établissent entre ces rapports eux-mêmes. Le récit d'une maladie qui emporte son personnage sera triste, mais celui d'une épidémie qui menace l'humanité risquera plutôt de basculer dans le thriller d'anticipation. Dans les deux cas le rôle, voire les objectifs de la médecine s'avèreront tout à fait différents, même si les données (corps ; maladie ; cure), et la violence (souffrance ; menace ; mort) restent les mêmes. Voilà un premier résultat découlant de la notion de proportion : la violence n'a plus besoin d'être départagée et de choisir son camp entre celui de l'esprit et celui du corps, entre

\footnotetext{
${ }^{2}$ Alexandre Gefen, « Le projet thérapeutique de la littérature contemporaine française », Contemporary French \& Francophone Studies, 20 :3, 2016, p. 420-427. Frédéric Worms, Revivre: Eprouver nos blessures et nos ressources, Paris, Flammarion, coll. «champs essais », 2015 [2012]; Emmanuel Bouju \& Alexandre Gefen (dir.), L'émotion, puissance de la littérature?, Bordeaux, Presses Universitaires de Bordeaux, 2013 ; JeanCharles Darmon, Littérature et thérapeutique des passions : La catharsis en question, Paris, Hermann, 2011 ; Sandra Laugier (dir.), Éthique, littérature, vie humaine, Paris, PUF, 2006.
} 
la représentation et la guérison. Notons qu'il n'y a rien là de particulièrement neuf, puisque ce n'est qu'un faible écho des conceptions philosophiques fameusement irréconciliables de Descartes et de Spinoza : la première envisageant les deux substances distinctes du corps et de l'esprit, la seconde une substance unique et infinie ${ }^{3}$. Mais comment réarticuler concrètement ce rôle de la proportion avec la démarcation restreinte et spécifique que nous avons identifiée entre la littérature et le domaine médical?

Il faut revenir à notre définition de la médecine telle que celle-ci concerne le maintien de l'équilibre des corps. La médecine a depuis longtemps rencontré un point commun avec la littérature grâce à l'idée harmonieuse de thérapie. Ce que partagent le travail du médecin et celui de l'écrivain est le souci de maintenir l'individu dans une homéostasie aux frontières statistiques mais néanmoins efficace. En ce sens la thérapie est ce qui ramène, et maintient, le patient dans le cercle de ce qui est compréhensible et communicable pour lui et pour les autres. C'est à cela qu'on se réfère d'abord en parlant de la santé. Les limites de cette homéostasie peuvent varier quelque peu. Mais ses dimensions - plus précisément: ses proportions - doivent rester compréhensibles. Comme l'établissait George Canguilhem : « normal et pathologique n'ont aucun sens à l'échelle où l'objet biologique est décomposé en équilibres colloïdaux et en solutions iodisées. » (Canguilhem, 2013, p. 86) La normalité de la santé n'est jamais donnée, mais elle devient vite partagée dès qu'on accepte la convention de proportions qui la fonde. Ainsi du système décrit jusqu'ici où littérature et médecine trouvent leur équilibre en s'échangeant le dicible et l'indicible de la violence. Autant

\footnotetext{
${ }^{3}$ Antonio Damasio a eu une influence importante dans la reconduction de cette opposition classique au sein du discours sur la médecine contemporaine. Antonio Damasio, Descartes' Error. Emotion, Reason, and the Human Brain, New York, Penguin, 1994 ; Antonio Damasio, Looking for Spinoza. Joy, Sorrow, and the Feeling Brain, New York, Harcourt, 2003.
} 
l'individu qui ne lit pas ni n'écrit est en santé du moment que son corps s'épanouit, autant l'écrivain qui écrit est lui aussi en santé même si son corps est malade. Et quand l'écriture et la parole ne tiennent plus, la médecine intervient dans sa pratique soit pour rétablir l'homéostasie, soit pour annoncer la mort.

C'est précisément cela qui s'est brisée à notre époque de systématicité accrue, cette harmonie de l'individu littéraire et du maintien de la santé. Pourquoi ? Parce que la médecine dorénavant, on l'a dit, c'est avant tout le système de santé. Différence non négligeable pour celui qui en devient le sujet, ou l'objet. Suivant cela la réponse à la question posée en ouverture, à savoir s'il existe des objets dans le domaine de la santé dont les proportions les rendent insaisissables pour la pensée littéraire, serait oui. Du moins si on accepte de suivre la leçon prodiguée par les philosophes néo-réalistes comme Bruno Latour (2011) avec son principe d'irréduction, Quentin Meillassoux (2013) avec ses fictions hors-sciences, et surtout Timothy Morton (2013) avec son concept d'hyper-objet. Mais il faut ajouter que ces objets disproportionnés n'appartiennent pas davantage - n'appartiennent plus devrions-nous dire aux mondes de la science. L'hyper-objet proposé par Timothy Morton l'illustre le mieux. Il existe en l'absence du monde qu'il a lui-même contribué à faire disparaître. Il n'est appréhensible que par ses effets. Et il semble « exister dans un système causal à la Hume où nous n'avons que des associations, des corrélations, et des calculs probabilistes pour nous appuyer. » (Morton, 2013, p. 39. Je traduis) Dans ce contexte il n'y a, derechef, plus de démarcation entre la littérature qui fouille l'âme et la médecine qui prend son relais quand les choses tournent mal.

Qui plus est, l'indicible se voit également invalidé. La médecine comme système et comme gestion est en passe de devenir elle-même un hyper-objet dont les proportions sont 
www.revue-analyses.org, vol. 14, n², automne-hiver 2019, p. 20-37.

incompatibles avec le récit des souffrances corporelles de l'individu. La pensée littéraire doit s'adapter à ce nouvel état de fait. C'est le cas par exemple avec le roman très remarqué de Maylis de Kérangal, Réparer les vivants, en 2014, qui est la mise en scène d'une transplantation cardiaque sans héros particulier, dont le personnage principal, si l'on veut, se situerait quelque part dans tout ce qui circule entre le corps atomisé du donneur et le corps en suspens du bénéficiaire. Plutôt qu'un projet esthétique comme ce pouvait être le cas avec les avant-gardes, c'est désormais la notion de proportion elle-même qui peut entraîner le dépassement des formes d'écriture focalisées sur le personnage et les structures narratives organisées autour de la pensée individuelle afin de créer une proximité inédite entre le monde fictionnel et l'expérience de la réalité. L'importance du microscopique et du macroscopique devient capitale dans les œuvres aujourd'hui à même de faire ressentir un tel vertige.

\section{L'exemple de Heather O'Neill et de Rawi Hage}

Afin de mieux illustrer ce qui précède, passons à deux brefs exemples de romans dont l'action se déroule à Montréal. Au lieu de s'attacher à la pratique médicale au sens traditionnel, ces romans font se dessiner l'ombre d'un système de gestion de la santé qui infiltre les vies et les influencent jusque dans leurs formes. Ces deux romans québécois publiés en 2006 et 2008 sont Lullabies for Little Criminals de Heather O’Neill et Cockroach de Rawi Hage.

Dans le premier, une enfant grandit entre l'univers interlope de son père junkie et celui des maisons d'accueil pour délinquants d'âge mineur en périphérie de Montréal. Pour survivre, elle ne se raconte pas des histoires d'évasion ni de bonheur romantique. Elle essaie plutôt de mesurer le degré d'adéquation des autres enfants des centres d'accueil et de la rue avec les idées de «crime» et de «criminel» auxquelles elle s'identifie sans rien y comprendre. Le résultat est charmant, en même temps que tout à fait sinistre. Difficile de 
parler de résilience à propos de la petite héroïne de O'Neill car son système de compréhension du monde est basé sur un mélange d'ignorance et de fabulation dont les proportions, si elles venaient à changer, pourraient tout faire se rompre. Baby (c'est son nom) n'a pas l'impression qu'elle est malade, physiquement ou socialement. Et pas non plus qu'elle est prise en charge par un système gestionnaire et aveugle, malgré que ça soit absolument le cas. Qui plus est, elle se trouve mieux à la fin du roman qu'au début, et ce malgré son père qui meurt d'overdose devant elle. Elle n'est pas mieux parce qu'elle aurait « intégré » la société. Ni parce qu'elle aurait adopté un code éthique différent. Elle est mieux parce qu'à force de temps, elle a compris le système de santé dans lequel sa vie est prise. Comment cette compréhension s'exprime-t-elle ? Dans la réalisation qu'il n'existe pas de hiérarchie entre les choses, mais seulement des proportions. Les centres d'accueil se valent (les géographies urbaine et périurbaine de Montréal sont d'ailleurs à peine présentes dans le récit), les souffrances s'amplifient ou diminuent suivant des causes incongrues, et les joies clignotent au gré des circonstances. Heather O’Neill a moins écrit un roman sur la « dure réalité de la rue » qu'elle n'en a écrit un sur la préadolescence comme un univers dont les proportions peuvent, sans les équivaloir, à tout le moins résister à celles de l'appareil de gestion de l'hygiène sociale. Lullabies for Little Criminals est un livre habité par une espèce de joie spinoziste.

Le second roman s'ouvre sur le dilemme d'un immigrant libanais anonyme qu'un suicide raté a conduit, par décret de la cour, dans le cabinet d'une psychiatre de Côte-desNeiges pour une thérapie payée par le gouvernement provincial. Dans la scène d'ouverture, il ne se demande plus du tout s'il devrait vivre ou mourir, mais plutôt s'il devrait ou non voler le rouge à lèvre dans le sac à main de la psychiatre ; ce qu'il fait deux pages plus loin avant 
www.revue-analyses.org, vol. 14, n², automne-hiver 2019, p. 20-37.

de sortir dans la rue enneigée. Cockroach de Rawi Hage est un monologue intérieur auquel on ne peut guère prêter confiance tant le personnage nous semble de mauvaise foi. Mais on s'aperçoit bientôt que cela n'a aucune importance. La vraie matière du texte tient aux conditions dans lesquelles le personnage est contraint de ne s'adresser qu'à lui-même. Il habite la grande pauvreté de Montréal avec une logique de guerre civile : rapt, butin, troc, cyclothymie émotionnelle, méfiance, bouffées de camaraderie disproportionnées. Il désire toutes les femmes qu'il rencontre indépendamment de leur statut; il n'a pas d'égard pour les conventions de politesse, et encore moins pour la propriété privée puisqu'il s'imagine en coquerelle rampant la nuit chez les gens (chez sa psychiatre surtout) afin d'y dérober des objets. Au bout d'un certain temps, on se rend compte que ces incursions nocturnes ont lieu pour vrai. On se rend compte d'une autre chose aussi, une chose qui est peut-être la vraie force de l'écriture par ailleurs très violente de Rawi Hage. À l'encontre de son impression initiale, le lecteur se rend compte, un peu choqué, que la mauvaise foi est, précisément, ce qui fait défaut à l'univers de Cockroach. Tout ce qui s'y dit est dit au premier degré. Il n'y a pas d'arrière-pensées.

Encore une fois, la leçon est qu'il n’y a pas de hiérarchie entre les choses, seulement des proportions. Mais Rawi Hage complexifie l'affaire. Le retour réflexif qui est la prérogative du lecteur n'existe pas sur un autre plan plus élevé et plus calme où la pensée accueillerait le contenu du roman comme la représentation de telle ou telle violence, de telle ou telle injustice à réparer. Il n'a pas d'autre plan. Il n'y en pas la possibilité. Les minuscules tours et détours sordides empruntés de nuit dans les logements des autres par l'être-coquerelle fantasmé en avatar du narrateur sont certes considérablement plus petits que les ramifications opaques du système bureaucratique de santé mentale qui s'occupe de son destin civique à 
Montréal. Mais, et c'est là toute la nature de la violence du roman, ils ne sont pas d'une autre nature. Ni l'un ni l'autre n'instaure de séparation entre l'esprit déclaré malade et le corps potentiellement criminel du protagoniste. Partant, ils ne constituent pas des façons fondamentalement différentes d'engager le corps déclaré malade sur le chemin de sa guérison.

Le personnage de Cockroach est-t-il mieux à la fin du livre ? La question n'a cette fois pas de sens. Les critères du « mieux » sont produit par le système de santé qui l'a pris en charge et qui n'est pas meilleur ni moins bien, mais juste plus vaste et en rapport avec d'autres hyper-objets comme par exemple l'idée de "population migrante» ou de «patients en réhabilitation ». Le personnage, lui, n'a pas autre chose que son discours autocentré dépourvu de filtre et la mémoire de la guerre (elle-même un hyper-objet) qui l'a vu naitre. Le livre se referme sur une scène de grande violence mais sans catharsis aucune ; un couteau est brandi, des coups de feu sont tirés, du sang est répandu. Or le personnage pourrait hésiter entre aller se pendre ou prendre un café à la page suivante, s'il y en avait une, que nous n'en serions pas surpris outre-mesure. Pourtant il n'est pas fou, ni amoral. De fait, dans les deux romans donnés en exemple la violence n'est pas excusée par un nihilisme ou une relativisation des valeurs tous azimuts. Ce sont des romans violents parce qu'ils nous forcent à regarder par un seul bout de la lorgnette : celui de l'individu et de ses proportions qui ne correspondent plus à celles du monde de la santé physique ou mentale. On peut dire, sans exagérer, que la société n'existe pas dans les livres de O'neill et de Hage. Des jeux de proportions monstrueux l'ont remplacée ; et ils ont également remplacé le destin «biographique» traditionnel des personnages. Le seul dénominateur commun qui subsiste est que tout cela demeure médicalisable, labelisable, institutionalisable. Le corps de Baby, dans une scène effarante, 
est détaillé à partir des gestes qu'elle croit devoir faire en se prostituant. Quant à Cockroach, il résiste à la déconstruction psychanalytique en se donnant comme on l'a vu les traits physiques d'un cloporte. C'est horrible, certes, mais pas étranger à la logique de leur monde. Lullabies for Little Criminals et Cockroach sont des histoires où la violence des proportions se substitue à l'ordre rassurant de la santé littéraire selon laquelle le corps et l'esprit n'ont pas besoin d'être réunis dans l'expérience de l'écriture ou de la lecture. Chez O'Neill et Hage, le corps et l'esprit au contraire ne peuvent pas être séparés. Ce sont deux textes dont les raisons premières et dernières tiennent dans les mesures variables entre le corps littéraire d'un personnage et le corps institutionnalisé d'un patient.

Pour conclure on ajoutera qu'il faut éviter d'appeler résilience l'inconscience de leur condition sociale « pathologique » chez les personnages de O’Neill et Hage. Cela est resté informulé jusqu'ici, mais il apparaît que cette inconscience entre en conflit avec les éthiques du care dont on aurait pu attendre la caution théorique afin d'appuyer l'ensemble des considérations précédentes ${ }^{4}$. Georges Canguilhem, encore une fois, peut nous aider à trouver l'ouverture qui manque dans ce cas entre résilience et éthique du care. Dans Le normal et le pathologique, il écrit:

Le normal, en matière biologique, ce n'est pas tant la forme ancienne que la forme nouvelle, si elle trouve les conditions d'existence dans lesquelles elle paraitra normative, c'est-à-dire déclassant toutes les formes passées, dépassées, et peut-être bientôt trépassées. (Canguilhem, 2013, p. 120)

Il y a dans cette remarque l'idée d'un corps non seulement en partie affranchi de son milieu, mais aussi affranchi de son histoire individuelle. La normalité médicale (la santé) n’est pas

\footnotetext{
${ }^{4}$ Voir par exemple les ouvrages-synthèse : Marian Barnes, Tula Brannelly, Lizzie Ward, \& Nicki Ward (dir.), Ethics of Care : Critical Advances in International Perspective, Bristol, Policy Press, 2015 ; Patricia Paperman \& Sandra Laugier, Le souci des autres : Éthique et politique du care, Paris, EHESS, 2011.
} 
le métadiscours qui correspondrait avec le récit de soi en littérature. Il y a des moments de « crise », dans nos vies individuelles, qu'on peut réarticuler à cette lumière, c'est-à-dire à la lumière des changements perçus comme violents dans la santé mais qui correspondent en vérité à des fluctuations (et non des crises) dans le processus infini d'adaptation du vivant en tant qu'il s'humanise en nous. Toutefois ce qui était net pour Canguilhem au niveau du vivant et de la «matière biologique » entraîne une série de questions pour nous. Dans la narration de soi, ces bouleversements proportionnels de la santé influencent comment la mécanique du récit? Sont-ils seulement compatibles avec l'histoire personnelle ? Et enfin, nos organes et les différentes homéostasies qui constituent notre organisme - car il y en a plusieurs - ontils quoi que ce soit de racontable, puisqu'ils n'existent pas suivant la même temporalité ? Le dicible et l'indicible qui ont servi au début de ce texte à souligner comment notre conception classique de la littérature s'accommodait de la violence avec l'appui de la santé était en fait une opposition de surface. On voit maintenant que la véritable distinction d'échelle entre la violence propre au littéraire et la violence propre au médical est tout simplement l'idée de biographie, c'est-à-dire le besoin que nous avons d'un récit de vie minimal, d'un cadre narratif pour concevoir le soi. Besoin qui pour la pensée littéraire donne la véritable mesure de son harmonisation classique avec la santé. En effet ramener vers le mieux, dans ce cadrelà, signifie ramener à la littérature, oui, mais plus fondamentalement à la biographie : c'està-dire à l'assurance que notre intelligence narrative, dont tant de valeurs découlent, n'est pas atteinte. Or devant les hyper-objets de notre contemporanéité, et devant le fait qu'il nous faut en toute honnêteté penser ceux-ci sur le plan de nos vies, il est permis de craindre que cette époque de la « santé littéraire » ait pris fin. 
www.revue-analyses.org, vol. 14, n², automne-hiver 2019, p. 20-37.

\section{Bibliographie}

BARnes, Marian, Tula Brannelly, Lizzie WARD, \& Nicki WARD (dir.) (2015), Ethics of Care : Critical Advances in International Perspective, Bristol, Policy Press.

BouJu, Emmanuel \& Alexandre GEFEN (dir.) (2013), L'émotion, puissance de la littérature?, Bordeaux, Presses Universitaires de Bordeaux.

CANGUILHEM, Georges, (2013 [1950]), Le normal et le pathologique, Paris, PUF.

CASEY, Nel (dir.) (2002), Unholy Ghost : Writers on Depression, New York, Perennial.

DAmasio, Antonio (1994), Descartes' Error. Emotion, Reason, and the Human Brain, New York, Penguin.

Damasio, Antonio (2003), Looking for Spinoza. Joy, Sorrow, and the Feeling Brain, New York, Harcourt.

DARMON, Jean-Charles (2001), Littérature et thérapeutique des passions : La catharsis en question, Paris, Hermann.

De KÉRAngAL, Maylis (2014), Réparer les vivants, Paris, Verticales.

GEFEN, Alexandre (2016), « Le projet thérapeutique de la littérature contemporaine

française », Contemporary French \& Francophone Studies, 20 :3, p. 420-427.

HaGe, Rawi (2008), Cockroach, Toronto, House of Anansi Press.

JUDT, Tony (2010), The Memory Chalet, New York, Penguin.

LATOUR, Bruno (2011 [1984]), Pasteur : guerre et paix des microbes, suivi de Irrédctions, Paris, La Découverte.

LAUGIER, Sandra (dir.) (2006), Éthique, littérature, vie humaine, Paris, PUF.

MeILlassoux, Quentin (2013), Métaphysique et fiction des mondes hors-science, Paris, Éditions Aux Forges de Vulcain.

Morton, Timothy (2013), Hyperobjects. Philosophy and Ecology After the End of the World, Minneapolis, University of Minnesota Press.

NANCY, Jean-Luc (2003), Au fond des images, Paris, Galilée.

OGIEN, Ruwen (2017), Mes mille et une nuits : La maladie comme drame et comme comédie, Paris, Albin Michel. 
O’NeILL, Heather (2006), Lullabies for Little Criminals, Toronto, Harper Perrenial.

PAPERMAN Patricia et Sandra LAUGIER (2011), Le souci des autres : Éthique et politique du care, Paris, EHESS.

Solomon, Andrew (2001), The Noonday Demon : An Atlas of Depression, New York, Scribner.

ST-GERMAIN, Philippe (2017), La greffe de tête, Montréal, Liber.

Styron, William (1990), Darkness Visible : A Memoir of Madness, New York, Vintage.

WeIL, Simone (1999), Euvres, Gallimard, coll. «Quarto ».

WORMS, Frédéric (2015 [2012]), Revivre : Éprouver nos blessures et nos ressources, Paris, Flammarion, coll. « champs essais ».

\section{Notice biobibliographique}

Daniel Laforest est professeur titulaire à l'Université de l'Alberta. Ses recherches actuelles portent sur les rapports entre littérature et humanités médicales. Il est auteur de $L$ 'archipel de Caïn. Pierre Perrault et l'écriture du territoire (XYZ, prix Jean-Éthier-Blais 2011), et de L'âge de plastique. Lire la ville québécoise contemporaine (Presses de l'Université de Montréal 2016). Il a également co-dirigé Literary Narrative, the Biomedical Body, and Citizenship in Canada (PULIM 2016, avec G. Clermont et B. Rouby), et Inhabiting Memory in Canadian Literature (UofA Press 2017, avec B. Authers et M. Snauwaert). Il a été chercheur titulaire de la Chaire en études canadiennes aux Université de Poitiers et de Limoges, ainsi que professeur invité du programme Medicine and the Muse au Center for Biomedical Ethics de l'Université Stanford aux États-Unis. 